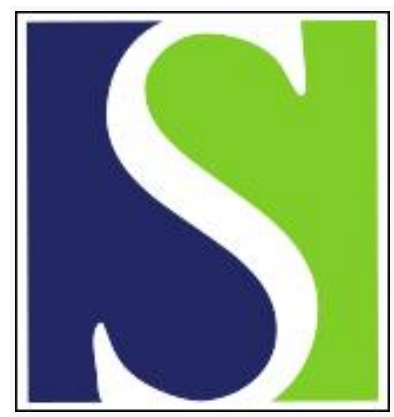

Scand J Work Environ Health 2017;43(2):181-186

https://doi.org/10.5271/sjweh.3613

Published online: 14 Dec 2016, Issue date: 01 Mar 2017

Occupational self-coding and automatic recording (OSCAR): a novel web-based tool to collect and code lifetime job histories in large population-based studies

by De Matteis S, Jarvis D, Young H, Young A, Allen N, Potts J, Darnton A, Rushton L, Cullinan P

The traditional manual collection and coding of lifetime job histories is unfeasible in large epidemiological studies. To overcome this, we have developed OSCAR, a new, validated, web-based tool that enables participants to self-collect and code their lifetime job-history efficiently. OSCAR could become the new standard approach to collect and code lifetime occupational exposures in large epidemiological studies.

Affiliation: Imperial College London, National Heart \& Lung Institute, Respiratory Epidemiology, Occupational Medicine and Public Health, Emmanuel Kaye Building, 1b Manresa Road, London SW3 6LR, UK. s.de-matteis@imperial.ac.uk

The following article refers to this text: $2023 ; 49(1): 53-63$

Key terms: automatic recording; data coding; data collection; exposure assessment method; lifetime job history; occupation; occupational self-coding; OSCAR; population-based study; standard occupational classification; web-based tool

This article in PubMed: www.ncbi.nlm.nih.gov/pubmed/27973677

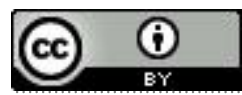




\title{
Occupational self-coding automatic recording (OSCAR): a novel web-based tool to collect and code lifetime job histories in large population-based studies
}

\author{
by Sara De Matteis, PhD, ${ }^{1}$ Deborah Jarvis, MD, ${ }^{1}$ Heather Young, PhD, ${ }^{2}$ Alan Young, PhD, ${ }^{2}$ Naomi Allen, \\ PhD, ${ }^{2}$, James Potts, BSc, ${ }^{1}$ Andrew Darnton, PhD, ${ }^{3}$ Lesley Rushton, PhD, ${ }^{4}$ Paul Cullinan, $M D^{1}$
}

\begin{abstract}
De Matteis S, Jarvis D, Young H, Young A, Allen N, Darnton A, Rushton L, Cullinan P. Occupational self-coding and automatic recording (OSCAR): a novel web-based tool to collect and code lifetime job histories in large populationbased studies. Scand J Work Environ Health. 2017;43(2):181-186. doi:10.5271/sjweh.3613
\end{abstract}

\begin{abstract}
Objectives The standard approach to the assessment of occupational exposures is through the manual collection and coding of job histories. This method is time-consuming and costly and makes it potentially unfeasible to perform high quality analyses on occupational exposures in large population-based studies. Our aim was to develop a novel, efficient web-based tool to collect and code lifetime job histories in the UK Biobank, a population-based cohort of over 500000 participants.
\end{abstract}

Methods We developed OSCAR (occupations self-coding automatic recording) based on the hierarchical structure of the UK Standard Occupational Classification (SOC) 2000, which allows individuals to collect and automatically code their lifetime job histories via a simple decision-tree model. Participants were asked to find each of their jobs by selecting appropriate job categories until they identified their job title, which was linked to a hidden 4-digit SOC code. For each occupation a job title in free text was also collected to estimate Cohen's kappa $(\kappa)$ inter-rater agreement between SOC codes assigned by OSCAR and an expert manual coder.

Results OSCAR was administered to 324653 UK Biobank participants with an existing email address between June and September 2015. Complete 4-digit SOC-coded lifetime job histories were collected for 108784 participants (response rate: $34 \%$ ). Agreement between the 4-digit SOC codes assigned by OSCAR and the manual coder for a random sample of 400 job titles was moderately good $[\kappa=0.45,95 \%$ confidence interval $(95 \% \mathrm{CI})$ $0.42-0.49]$, and improved when broader job categories were considered $(\kappa=0.64,95 \%$ CI $0.61-0.69$ at a 1 -digit SOC-code level).

Conclusions OSCAR is a novel, efficient, and reasonably reliable web-based tool for collecting and automatically coding lifetime job histories in large population-based studies. Further application in other research projects for external validation purposes is warranted.

Key terms data collection; data coding; exposure assessment method; occupation; standard occupational classification.

It has been estimated that globally in 2013 about 700000 deaths and 55 million disability-adjusted lifeyears (DALY) are attributable to occupational risks (1). Identification of high-risk jobs for workers' health is pivotal not only to identify the underlying occupational causal agents but also to implement focused preventive strategies aimed at eliminating or at least reducing exposures and so avoid the associated disease burden
(2). Large population-based epidemiological studies are the ideal setting to identify which occupations are associated with adverse health effects because of the broad variety of industries, jobs, and exposure levels found in a general population. However, an important limitation of such large studies is the cost of occupational exposure assessment. There is no standardized method to collect individual lifetime job histories in epidemiological

1 Department of Respiratory Epidemiology, Occupational Medicine and Public Health, National Heart and Lung Institute, MRC-PHE Centre for Environment and Health, Imperial College London, London, UK.

2 Clinical Trial Service Unit and Epidemiological Studies Unit, Nuffield Department of Population Health, University of Oxford, Oxford, UK.

3 Health and Safety Executive, Bootle, Merseyside, UK

4 Department of Epidemiology and Biostatistics, School of Public Health, MRC-PHE Centre for Environment and Health, Imperial College London, London, UK.

Correspondence to: Sara De Matteis, Imperial College London, National Heart \& Lung Institute, Respiratory Epidemiology, Occupational Medicine and Public Health, Emmanuel Kaye Building, 1b Manresa Road, London SW3 6LR, UK. [E-mail: s.de-matteis@imperial.ac.uk] 
studies but the most common approaches are: (i) faceto-face personal interviews, (ii) self-administered job questionnaires, (iii) retrieval of job/industry titles from contemporary and historical occupational records, or (iv) record linkage with national administrative registrybased employment data, such as those available in the Nordic Countries (3). These data, typically in narrative form, must then be translated into meaningful job/industry titles and subsequently converted into standard codes to be usable in statistical analyses. Traditionally, the most reliable approach is considered to be manual coding by trained staff who assign occupation and industry codes based on standard occupational classifications. This process is very time consuming (and expensive) and thus often unfeasible in large epidemiological studies. An alternative approach is the use of available automatic web tools (eg, CASCOT) (4), but the validity and reliability of these systems ranges from poor to uncertain and is greatly affected by the quality of the input job description data. Text entry errors, misspellings, non-standard abbreviations, transposed occupation and industry, and listing of multiple occupations and industries are examples of why some manual coding is nearly always required following use of an automated coding system (5). Furthermore, manual coders, even if trained, can only access second-hand information, frequently of low quality, and may have limited expertise in jobs in some industrial sectors resulting in important job misclassification or missing job coding.

To overcome the above limitations, a novel, efficient and reliable method to both collect and code occupational lifetime job histories is needed, combining the validity of manual standard coding with the efficiency of automatic software. Our aim was to develop a webbased tool named OSCAR (occupations self-coding automatic recording) to enable individuals to construct their own job history through a simple web interface, based on the premise that only the worker knows exactly what his/her job is and is therefore the best person to collect his/her lifetime job history.

\section{Methods}

\section{OSCAR development}

OSCAR was originally designed for the UK Biobank study (6), a large population-based cohort including over 500000 men and women aged 40-69 years recruited throughout the UK between 2006-2010, principally to estimate the burden of work-related chronic obstructive pulmonary disease (COPD). OSCAR can be used to investigate the association of occupational exposures with any health outcome.
We designed OSCAR not only to be easy and quick to use (with a target completion time of 20 minutes), but also reliable. To achieve this we developed a categorical decision tree based on a simplified but faithful version of the hierarchical structure of the UK Standard Occupational Classification (SOC), version 2000 , to create job categories to enable participants to quickly and easily find each job they have held. The UK Office for National Statistics (ONS) developed SOC (7) to classify all occupations in the UK general population into standard codes according to the following hierarchical structure: 9 major groups (1-digit SOC codes), 25 sub-major groups (2-digit SOC codes), 81 minor groups (3-digit SOC codes), and 353 unit groups (4-digit SOC codes); the higher the number of SOC digits, the greater the level of detail in the job definition (available at http://webarchive.nationalarchives.gov.uk/20160105160709/http://www.ons. gov.uk/ons/guide-method/classifications/archivedstandard-classifications/standard-occupational-classification-2000/index.html). SOC was developed for statistical purposes, and its language (eg, elementary trades and related occupations) is often technical and not easily understood without formal training. For these reasons, we undertook a comprehensive rewording of the job categories and titles to help participants recognize and self-allocate each of their jobs. In addition, we simplified the SOC hierarchical structure to a three-level decision tree displayed in OSCAR as job lists on three linked webpages starting with the major job groups (proxy for 1-digit SOC codes), followed by the job subgroups (proxy for 2- and 3-digit SOC codes) and ending in specific job titles (ie, the original 353 4-digit SOC codes). On selection of a final job title, a hidden 4-digit SOC code is automatically assigned to that job and retained in the database. Further, in the grouping of job categories, we did not follow the same education-based criteria used by SOC, but rather an analogy-based one: eg, engineer and engineer technician were grouped into the same major job group category (based on the similarity of their jobs) while in the original SOC 2000 they are categorized separately because of their different respective educational levels (professional versus technical) and were then distinguished at the final job-title level (eg, civil engineer coded as "2121" versus engineering technician as "3113"). All the original 353 4-digit SOC codes are included in OSCAR to ensure that almost all jobs in the UK general population are covered. There is no standard classification that can cover in detail all existing jobs, for which reason the SOC classification includes categories for jobs "not elsewhere classified". In instances where an exact job title could not be found, participants were asked to select the closest match. Because some jobs are intrinsically generic (eg, secretary) and are therefore found in different job sectors, we 
duplicated these job titles across different job categories to ensure that a participant could find his/her job easily. "Warning" and "hint" messages including examples of key jobs included in each job category were displayed as "tool tips" throughout the process to assist participants in their progression through the decision tree to the final job title. Finally, we used a color system to differentiate the major job groups (designed to be a proxy for industry sectors) and the subsequent job groupings, and job titles to help participants self-allocate the correct job while progressing through the three webpages.

\section{Implementation of OSCAR on the UK Biobank website}

OSCAR was implemented as a web-based platform and hosted by the Clinical Trial Services Unit (CTSU), at the University of Oxford, Oxford, UK. It should be noted that OSCAR is also compatible with any kind of electronic device (eg, phones, tablet devices).

An introductory screen informed Biobank participants about the OSCAR process and inclusion criteria for the collection of jobs as specified by our particular research requirements (ie, that participants only needed to consider all paid jobs held for $\geq 6$ months and for an equivalent of 15 hours per week since they left full-time education (school/college/university).

In order to internally validate OSCAR, we also asked participants to type their job title (in free-text format), as well as the year they started and ended each job; they then proceeded to find their job category for the specific job using the standard OSCAR process. We also asked them to enter dates of any gaps in their work history and the reasons for these (eg, maternity leave, unemployment, further education, etc.). A job-history timeline table was progressively built up as they entered each job, which allowed participants to visualize their job history over time, with the option to amend any entries at any time in the process. Participants were allowed to count several consecutive similar jobs for different employers as one (eg, a doctor at different hospitals).

Further submodules included questions for each job on the number of hours worked per week, shift patterns, and exposure to specific occupational agents. After completion of their job history, Biobank participants were asked to complete some questions on their respiratory health and smoking habits to address specific questions posed within our research project.

As an illustration of the overall process, an example of the selection of the job "bricklayer" using OSCAR is displayed in figures $1-4$.

First, the Biobank participant is asked to type in the job title and the corresponding year or age at the start and end of this job (figure 1). The participant is then invited to find the same job through a series of job lists; of note only one selection among the multiple job cat- egories displayed for each job held was allowed by the system. The first job list displayed on the webpage corresponds to the first SOC major job groupings (displayed as 15 colored boxes in figure 2). A red circle has been superimposed to indicate the most appropriate selection for the bricklayer job.

By selecting the major job grouping, the participant automatically progresses to the next webpage where more detailed job categories included in the construction job sector are displayed. Again, the participant would drill down to find the job title that is most suitable, in this case the first option (figure 3).

After this selection, the participant progresses to the final webpage where he/she will select the most suitable job title and, without being aware of the underlying coding, will automatically assign a 4-digit SOC code to the

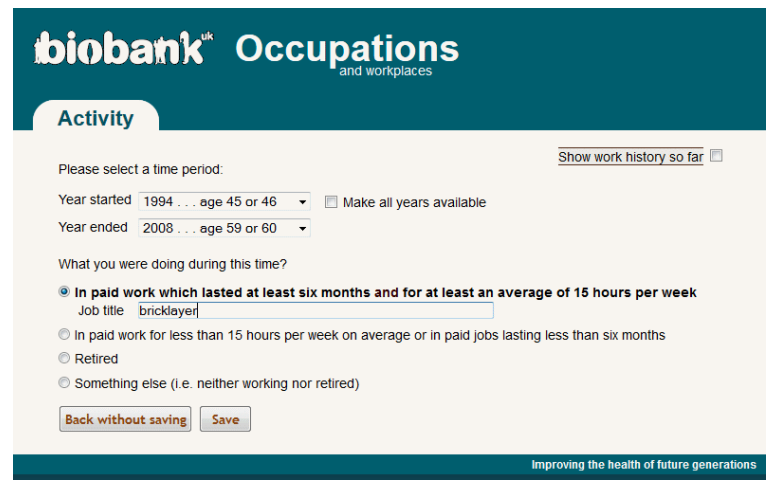

Figure 1. Job title entry webpage (bricklayer is used as an example).

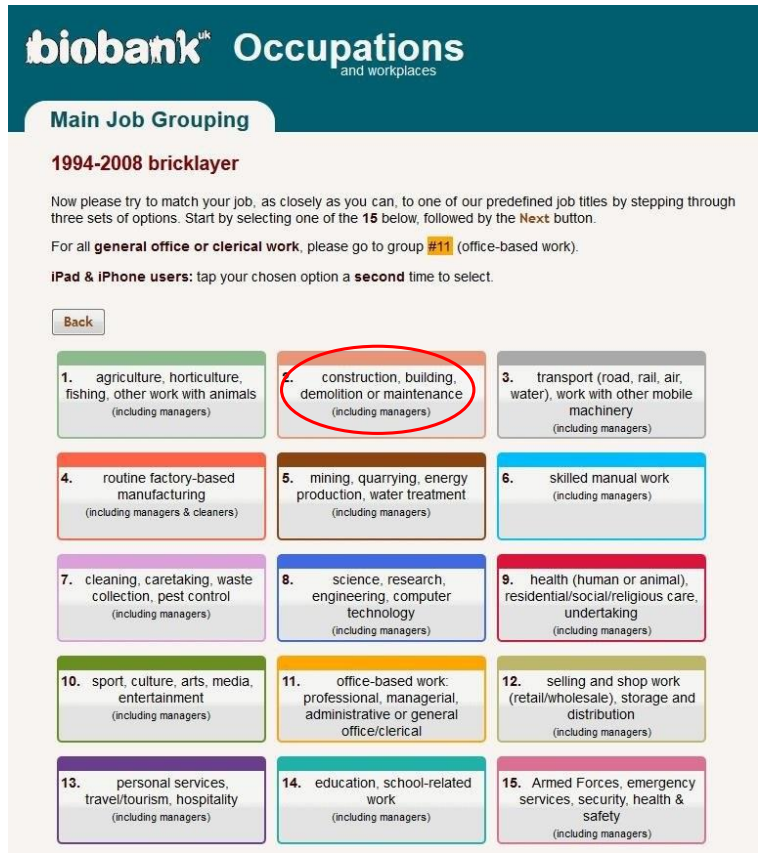

Figure 2. Major job groupings webpage for participants to select the most suitable job sector (construction sector is selected as an example). 


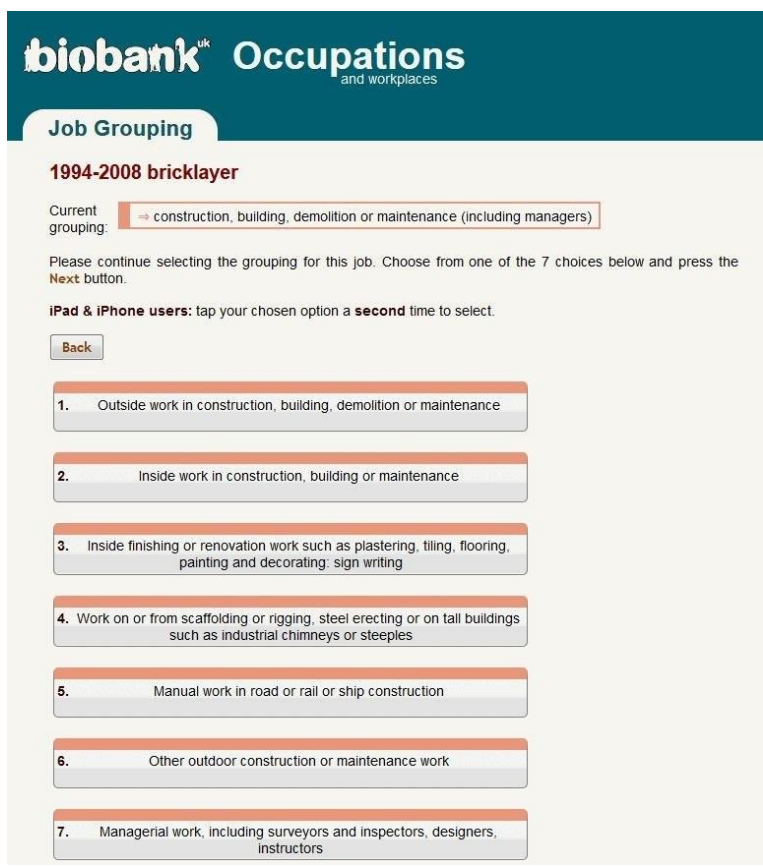

Figure 3. Job categories webpage (outside construction work is selected as an example).

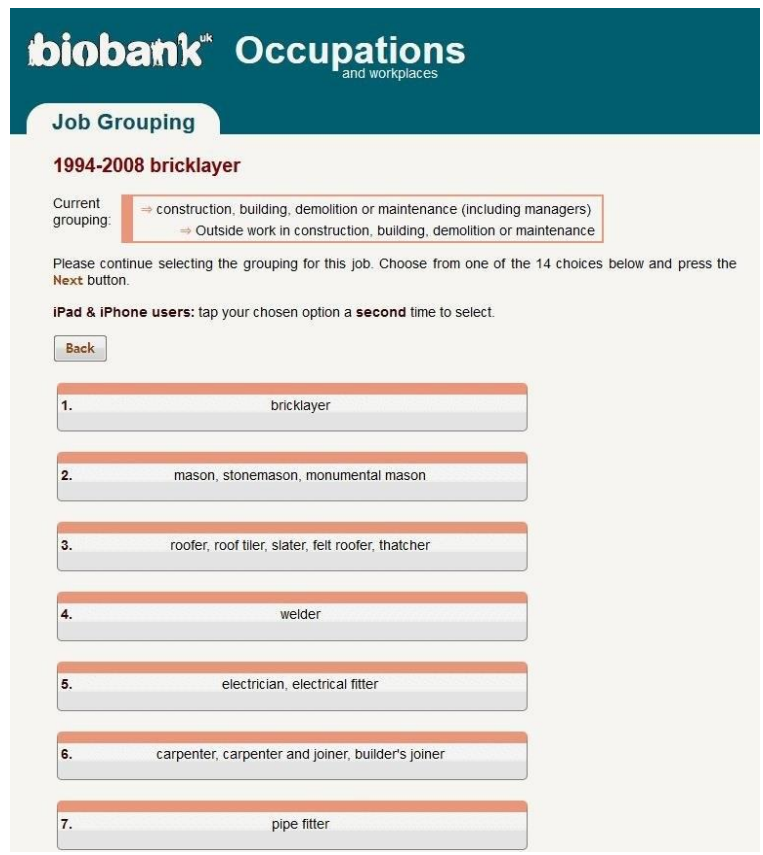

Figure 4. Final job titles webpage (bricklayer is selected as an example).

selected job (figure 4). The corresponding job-history timeline is displayed in figure 5 .

\section{OSCAR administration and validation}

OSCAR was administered by email to all UK Biobank participants with an available email address $(\mathrm{N}=324653)$ between June and September 2015; reminder emails

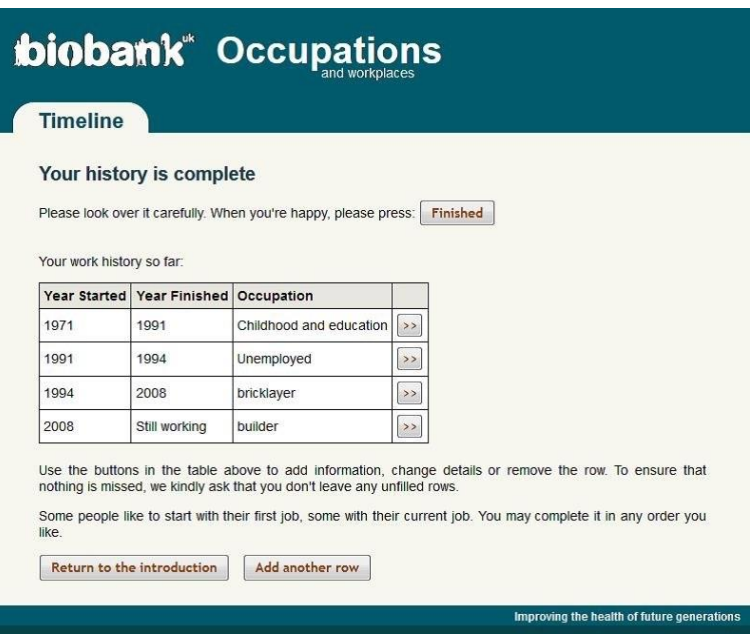

Figure 5. Timeline webpage (the bricklayer job-history timeline is displayed as an example).

were sent to partial- and non-responders up until December 2015. If needed, participants could telephone UK Biobank's Participant Resource Centre and access an online set of frequently asked questions.

Among the verified and complete responses only, we used the freetext job title provided by each participant to perform an internal validation of OSCAR. An expert occupational coder, who was blind to the OSCAR SOC coding and health status of participants, manually coded a random sample of 400 (based on power calculations) (8) job titles. The agreement between the expert- (reference) and OSCAR-derived SOC codes was measured by calculating the Cohen's Kappa $(\kappa)$ coefficient and biascorrected 95\% confidence intervals $(95 \% \mathrm{CI})$. Cohen's Kappa is considered a more robust quantitative measure of inter-rater agreement (or reliability) than simple $\%$ agreement calculation because it takes into account the agreement occurring by chance. The $\kappa$ values of agreement are often interpreted as follows: $>0.80$ (very good), $0.61-0.80$ (good), 0.41-0.60 (moderate), 0.21-0.40 (fair), and $<0.2$ (poor) (9). All analyses were performed using Stata version 14 (StataCorp LP, College Station, TX, USA).

\section{Results}

Administration and internal validation

Overall, 108784 UK Biobank participants, aged 45-81 years (63 years on average) at time of questionnaire administration, fully completed OSCAR (response rate: $34 \%$ ). The duration for completion was around $20 \mathrm{~min}$ utes [median 17 (interquartile range: 11-30) minutes]. Participants included, on average, three jobs throughout their job history (up to a maximum of 40). 
The agreement between the 4-digit SOC codes assigned by OSCAR and the manual coder was moderately good $(\kappa=0.45,95 \%$ CI $0.42-0.49)$. OSCAR performance improved, as expected, when considering coding agreement between broader job categories. In particular, the overall agreement increased at the 3-digit level to $52 \%(\kappa=0.52,95 \%$ CI $0.49-0.54)$, at the 2-digit level to $62 \%(\kappa=0.62,95 \%$ CI $0.59-0.63)$, and at the 1-digit level to $64 \%$ ( $\kappa=0.64,95 \%$ CI $0.61-0.69)$. We tried to estimate the $\kappa$ for each of the 3534 -digit SOC codes, but the few observations within each SOC code in our sample of 400 job titles prevented computing reliable estimates; the same applies at the 3-digit level. At the 2-digit level, considering SOC codes with $\geq 5$ observations, the highest agreement was for the "54-" job groups $(\kappa=0.76,95 \%$ CI $0.54-1.00)$ corresponding in the original SOC classification to "textiles, printing and other skilled trades", and the lowest for the "52-" ( $\kappa=0.11,95 \%$ CI $0.08-0.19)$ corresponding to the SOC "metal machining, fitting and instrument making trades". At the broader 1-digit level, the highest agreement was for the "2-" job-groups ( $\kappa=0.58,95 \%$ CI $0.57-0.63$ ) corresponding to the SOC "professional occupations", and the lowest for the "1-" ( $\kappa=0.26,95 \%$ CI $0.14-0.39)$, corresponding to "managers and senior officials". Of note, while OSCAR assigned a 4-digit SOC code to all the job titles reported by the participants, the manual coder was unable to code two jobs due to lack of sufficient information in the job title input by the participant. In some cases, OSCAR codes appeared more specific than those obtained through manual coding. For example, the job title "research scientist" was coded as the generic 4-digit SOC "2321" by the manual coder but by OSCAR as "2113" corresponding to the more specific job title "geophysicist".

\section{Discussion}

OSCAR is an innovative web-based tool that combines the efficiency of computer-based automatic software with the validity of standard occupational coding in order to ensure fast and reasonably reliable collection and coding of individual job histories.

The response rate to OSCAR was $34 \%$, which is similar to previous questionnaires administered to the UK Biobank cohort (eg, 33\% for the dietary 24-hour recall questionnaire) and was completed within the target time of 20 minutes. In contrast, manual coding of a subset of 400 job titles took approximately 16 hours, emphasizing the efficiency of OSCAR and its application in large population-based studies where alternative occupational exposure approaches would be unfeasible. The number of jobs included by each participant also suggests the completeness of the job-history collection process; in addition, the automatic coding method ensures no missing data in the coding phase.

The internal validation supports the reasonable reliability of this method. In the absence of an agreed gold standard, OSCAR has shown moderately good agreement with the expert manual coding approach that is traditionally considered the most reliable method. Such comparisons are, however, difficult to interpret, not only because of OSCAR's original approach, but also because the reported inter-rater agreement of available occupational exposure methods is quite broad, ranging from very poor to moderate $(2,3,10)$. Moreover, the innovative, underlying concept of relying directly on a workers' ability to self-collect and code their own job history, allows a level of information accuracy otherwise impossible with alternative methods (including expert manual coders) and suggests that, after further validation, OSCAR itself could become a potential new reference coder. When compared with occupational records, self-reported job histories have been previously shown to be reliable (11) and repeatable (12-14). What is novel about OSCAR is that workers themselves, when supported by easy and quick web-based tools, can direct their entire occupational assessment process in a more efficient and possibly more accurate way than that available through costly, trained interviewers and manual coders.

We developed the underlying decision tree of OSCAR to be flexible and adaptable to any research project. The web-based interface makes it easily accessible across many different personal devices and negates the need to install or download any special software. The inclusion/exclusion criteria for jobs collection can be amended if needed or additional submodules can be added to fit specific research goals (eg, studies on breast cancer risk among healthcare workers). In addition, the collected 4-digit SOC codes can be linked to job-exposure matrices to automatically translate each job title into potential exposure to specific occupational agents. Lastly, compatibility with smart devices can help maximize response rates among participants.

Some limitations should be acknowledged. First, in our random sample of 400 job titles, the validation of OSCAR both quantitatively and qualitatively could not cover all the 353 SOC codes, but there is no a priori reason to expect that certain categories of workers would be systematically better or worse than others in finding their jobs among the groupings displayed by OSCAR and that the performance of OSCAR should be biased towards or against specific occupations. Second, the level of detail of the 4-digit SOC codes means that some jobs occurring in a general population are not included, but this a caveat of all existing standard occupational classification systems. However, because detailed job 
titles are usually grouped into broader job categories for statistical analysis, this is not, in itself, a problem. Third, it is impossible to rule out some residual job misclassification, but this is likely to be non-differential with regard to health status not only because participants were not aware of the specific research health outcome, but also because information on jobs rather than specific occupational exposures were collected (3). Finally, OSCAR uses a UK-based standard classification, but SOC codes can be easily mapped onto other national and international classifications [eg, to ISCO-88(15)] by using Excel worksheets developed by the ONS Classifications and Harmonization Unit, and available at the following websites (http://webarchive.nationalarchives. gov.uk/20160105160709/http://www.ons.gov.uk/ons/ guide-method/classifications/archived-standard-classifications/standard-occupational-classification-2000/ index.html).

In conclusion, OSCAR is a novel, efficient and reasonably reliable web-based tool for collecting and automatically coding lifetime job histories in large population-based epidemiological studies. Further application of OSCAR in a broad variety of research studies and study populations is warranted to validate this efficient occupational assessment tool externally.

\section{Ethics approval}

The National Health Service National Research Ethics Service has given ethics approval to the UK Biobank (Ref 11/NW/0382).

\section{Acknowledgements}

This work was supported by contract $\mathrm{OH} 1511$ from the Health and Safety Executive (HSE). The content of this paper contains the views of the authors, and not necessarily those of HSE. The authors declare no conflict of interest.

\section{References}

1. Forouzanfar MH, Alexander L, Anderson HR, Bachman VF, Biryukov S, Brauer M, et al. Global, regional, and national comparative risk assessment of 79 behavioural, environmental and occupational, and metabolic risks or clusters of risks in 188 countries, 1990-2013: a systematic analysis for the Global Burden of Disease Study 2013. Lancet 2015;386(10010):2287-323. https://doi.org/10.1016/S01406736(15)00128-2.

2. Mannetje A, Kromhout H. The use of occupation and industry classifications in general population studies. Int J Epidemiol. 2003;32(3):419-28. https://doi.org/10.1093/ije/dyg080.
3. McGuire V, Nelson LM, Koepsell TD, Checkoway H, Longstreth WT, Jr. Assessment of occupational exposures in community-based case-control studies. Annu Rev Public Health. 1998;19:35-53. https://doi.org/10.1146/annurev. publhealth.19.1.35.

4. Warwick Institute for Employment Research. CASCOT: Computer-Assisted Structured Coding Tool [accessed in June 2016]. http://www2.warwick.ac.uk/fac/soc/ier/software/ cascot/

5. Patel MD, Rose KM, Owens CR, Bang H, Kaufman JS. Performance of automated and manual coding systems for occupational data: a case study of historical records. Am JInd Med. 2012;55(3):228-31. https://doi.org/10.1002/ ajim. 22005 .

6. Sudlow C, Gallacher J, Allen N, Beral V, Burton P, Danesh $\mathrm{J}$, et al. UK biobank: an open access resource for identifying the causes of a wide range of complex diseases of middle and old age. PLoS Med. 2015;12(3):e1001779. https://doi. org/10.1371/journal.pmed.1001779.

7. Office for National Statistics. Standard Occupational Classification 2000 [accessed in Jun 2016]. http://www.ons. gov.uk/aboutstatistics/classifications/archived/SOC2000/ index.html.

8. Sim J, Wright CC. The kappa statistic in reliability studies: use, interpretation, and sample size requirements. Phys Ther. 2005;85(3):257-68.

9. Cohen J. Weighted kappa: nominal scale agreement with provision for scaled disagreement or partial credit. Psychol Bull. 1968;70(4):213-20. https://doi.org/10.1037/h0026256.

10. Kromhout H VR. Application of job-exposure matrices in studies of the general population: some clues to their performance. Eur Respir Rev 2001;11:80-90.

11. Bourbonnais R, Meyer F, Theriault G. Validity of self reported work history. Br J Ind Med 1988;45(1):29-32. https://doi. org/10.1136/oem.45.1.29.

12. Brower PS, Attfield MD. Reliability of reported occupational history information for US coal miners, 1969-1977. Am JEpidemiol. 1998;148(9):920-6. https://doi.org/10.1093/ oxfordjournals.aje.a009718.

13. Ikin JF, Fritschi L, Sim MR. Reproducibility of survey results from a study of occupation-related respiratory health in the aluminum industry. Appl Occup Environ Hyg.2002;17(11):77482. https://doi.org/10.1080/10473220290096023.

14. Rona RJ, Mosbech J. Validity and repeatability of self-reported occupational and industrial history from patients in EEC countries. Int J Epidemiol. 1989;18(3):674-9. https://doi. org/10.1093/ije/18.3.674.

15. Elias P. Occupational Classification (ISCO-88). Paris: OECD Publishing.

Received for publication: 26 August 2016 